\title{
Fast Multi-Step Optimization with Deep Learning for Data-Centric Supercomputing
}

\author{
Tsuyoshi Ichimura \\ Earthquake Research Institute and \\ Department of Civil Engineering, \\ The University of Tokyo \\ Center for Advanced Intelligence \\ Project, RIKEN \\ Tokyo, Japan \\ ichimura@eri.u-tokyo.ac.jp \\ Muneo Hori \\ Research Institute for \\ Value-Added-Information \\ Generation, Japan Agency for \\ Marine-Earth Science and \\ Technology \\ Yokohama, Japan \\ horimune@jamstec.go.jp
}

\author{
Kohei Fujita \\ Earthquake Research Institute and \\ Department of Civil Engineering, \\ The University of Tokyo \\ Center for Computational Science, \\ RIKEN \\ Tokyo, Japan \\ fujita@eri.u-tokyo.ac.jp
}

Lalith Wijerathne

Earthquake Research Institute and

Department of Civil Engineering,

The University of Tokyo

Tokyo, Japan

lalith@eri.u-tokyo.ac.jp

\author{
Takuma Yamaguchi \\ Earthquake Research Institute and \\ Department of Civil Engineering, \\ The University of Tokyo \\ Tokyo, Japan \\ yamaguchi@eri.u-tokyo.ac.jp
}

\author{
Naonori Ueda \\ Center for Advanced Intelligence \\ Project, RIKEN \\ Tokyo, Japan \\ naonori.ueda@riken.jp
}

\begin{abstract}
Following the improvement in data acquisition technology and in analysis capability, we develop an optimization method for datacentric supercomputing, which can utilize large-scale data. Prior information of the solution of these optimization problems is often available, and the use of such information is expected to improve the efficiency of the optimization process and the accuracy of its solution. Targeting use on supercomputing environments such as Big Data and Extreme-scale Computing (BDEC) resources, we develop a fast parameter optimization method that combines datacentric fast simulation methods and deep learning. This method comprises multiple steps, i.e., an approximate solution is obtained in a short time using prior information on many compute nodes, and the approximate solution is refined using a small number of compute nodes. Thus, optimum solutions can be obtained in a short time based on the required accuracy, even on many compute-node environments without fast interconnects. The developed method is expected to provide useful knowledge for related optimization problems computed on distributed ubiquitous computing resources using large observation data obtained by the Internet of Things (IoT) and fifth-generation (5G) networks.
\end{abstract}

Permission to make digital or hard copies of all or part of this work for personal or classroom use is granted without fee provided that copies are not made or distributed for profit or commercial advantage and that copies bear this notice and the full citation on the first page. Copyrights for components of this work owned by others than ACM must be honored. Abstracting with credit is permitted. To copy otherwise, or republish, to post on servers or to redistribute to lists, requires prior specific permission and/or a fee. Request permissions from permissions@acm.org.

HP3C 2020, fune 27-29, 2020, Guangzhou, China

(C) 2020 Association for Computing Machinery.

ACM ISBN 978-1-4503-7691-4/20/06 . \$ \$15.00

https://doi.org/10.1145/3407947.3407949

\section{CCS CONCEPTS}

- Computing methodologies $\rightarrow$ Optimization algorithms; Parallel algorithms.

\section{KEYWORDS}

optimization, deep learning, data-centric supercomputing, highperformance computing

\section{INTRODUCTION}

Due to the recent developments of Internet of Things (IoT) and fifth-generation $(5 \mathrm{G})$ network technologies, as well as the improvement in analysis capability, the demand for data-centric supercomputing, where large-scale observation data is rapidly inputted into supercomputing environment for fast analysis, has increased (e.g., Big Data and Extreme-scale Computing [1]). Here large-scale data obtained by geographically distributed IoT instruments are dynamically allocated to distributed compute-node resources, and the new observation data together with the stored data is used to generate information with additional value (Fig. 1). This study focuses on parameter optimization by utilizing observation data, which is a type of data-centric supercomputing with large demand. This optimization has various applications, such as estimation of the internal structure of biomedical and structural problems or estimation of traffic status and assimilation of traffic inflow. The optimized parameter itself is useful, and it can also be employed for smart control of physical space. Thus, the achievement of a fast optimization method, which can utilize the large data obtained at an arbitrary point and arbitrary time by dynamically allocating ubiquitous computational resources is expected to make broad ripple effects to society.

The optimization shown in Fig. 1 corresponds to supercomputing conducting many cases of $10^{0-1}$-second simulations using $10^{3-4}$ 


\section{Physical space}

loT with $5 \mathrm{G}$ network enables acquiring high accuracy/small quantity data and low accuracy/large quantity data, together with large volume data (e.g. videos)

\section{Measurement}

Big Data \& Extreme-scale Computing System

Analysis/estimation/prediction (based on mathematical/ computational/ computer science, especially highperformance computing and data science)

Figure 1: An integrated physical-virtual assimilation platform for computing various flowing data. Using ubiquitous data and computational resources, information is generated and physical space is controlled by assimilation (i.e., optimization of some kinds of distances) of physical space and virtual space. Obtaining sufficient optimization results in a short time is the key to achievement of this platform.

compute nodes for various optimization problems. In this paper, we target one trial of this optimization using $10^{3-4}$ compute nodes within $10^{0-1}$ seconds. Here, we develop a fast and efficient parameter optimization method applicable for a wide variety of optimization problems by combining fast simulation and deep learning. The parameter optimization method is designed to be multi-step, such that the needs for both obtaining approximate solutions in a very short time and obtaining a refined solution based on the accuracy requirements are satisfied. As the developed method is applicable to a variety of problems, we describe the method in a generalized form in Section 2. Following the description in Section 2, we show application of the method to a specific problem setting, and demonstrate its effectiveness in Section 3. In particular, we conduct a numerical experiment on an internal structure optimization problem using image-based wave propagation modeling. Instead of using the proposed method, conventional optimization approaches using large number of nodes in parallel (e.g., using our SC18 Gordon Bell Prize Finalist wave propagation solver [2] scalable up to full Summit [3]) can be used; however, the developed optimization method can extract prior information efficiently using deep learning and thus is capable of acquiring the optimized solution more robustly in a shorter time. For example, in the application problem in Section 3, a very good solution is estimated in very short time using only 2048 samples out of the prior knowledge data base of 470775 samples with two-step sampling (sampling rate of $0.00435(=2048 / 470775)$ ). Summarizing the above, the paper is concluded in Section 4. $\overline{\text { Algorithm } 1 \text { Fast multi-step optimization algorithm using deep }}$ learning. Lines 3 and 6 comprising most of the computational cost can be computed in parallel on a distributed supercomputing environment.

1: Input: dataset which consists of $n(\gg 1)$ samples of $\mathbf{m}_{i}$ that are close to the solution $\mathbf{m}^{\text {ref }}$

2: dataset $_{0} \Leftarrow$ Randomly extract $n_{0}(\ll n)$ samples from dataset

3: Compute $\operatorname{ERR}\left(\mathbf{m}_{i}\right)$ for all samples in dataset $_{0}$

4: Generate a classifier $D L_{a}$ that classifies the error level $E R R$ given $\mathbf{m}$ (training data: datase $_{0}$ )

5: dataset $_{1} \Leftarrow$ Extract $n_{1}(\ll n)$ samples that are classified to be of lowest error level from dataset using $D L_{a}$

6: Compute $\operatorname{ERR}\left(\mathbf{m}_{i}\right)$ for all samples in dataset $_{1}$

7: Generate a regression model $D L_{b}$ mapping $\mathbf{m}$ to $E R R$ (training data: samples in dataset $_{1}$ and dataset t $_{0}$ that are classified to be in the lowest error level)

8: m $^{\text {coarse }} \Leftarrow$ Minimize Eq. (2) based on $D L_{b}$ using $n_{m}$ samples with lowest error in dataset t $_{0}$ and dataset ${ }_{1}$

9: $\mathbf{m}^{\text {fine }} \Leftarrow$ Refine $\mathbf{m}^{\text {coarse }}$ using gradient based optimization scheme

\section{METHOD}

In this study, we design an optimization method for a data-centric supercomputing environment. It is often the case that there are some prior knowledge on the unknown target parameter, $\mathbf{m}$; that is, data for the target problem is accumulated and will increase in the future. Thus, we suppose that we have access to a database dataset with $n(\gg 1)$ candidate solutions $\mathbf{m}_{i}$. These solutions are close to the target solution, $\mathbf{m}^{r e f}$, but are not exactly the same as $\mathbf{m}^{r e f}$. Suppose a forward analysis for a set of model parameters $\mathbf{m}$ is defined as follows:

$$
\mathbf{u} \Leftarrow \mathbf{f}(\mathbf{m}) .
$$

We estimate $\mathbf{m}^{r e f}$ such that ERR of some form defined as the difference between the response $\mathbf{u}$ and the observation recorded $\overline{\mathbf{u}}$ is minimized. Hereon, we denote $E R R$ for $\mathbf{m}$ as $E R R(\mathbf{m})$. We consider a problem that requires a non-negligible cost to evaluate Eq. (1) with numerous local optimums in the minimization problem. This problem often leads to local solutions when using gradient-based optimization methods. Using ubiquitously distributed large-scale computing resources for such problem, we design a method that can estimate a solution $\mathbf{m}^{\text {coarse }}$ close to $\mathbf{m}^{\text {ref }}$ in a very short time, and this is capable of solution refinement that can make the solution $\mathbf{m}^{\text {fine }}$ closer to $\mathbf{m}^{\text {ref }}$. Below we explain the developed optimization method following Algorithm 1.

First, we consider how to obtain an approximate solution $\mathbf{m}^{\text {coarse }}$ in a short time. Since a database with numerous candidate solutions is available, one way to obtain $\mathbf{m}^{\text {coarse }}$ is to choose $\mathbf{m}_{i}$ with the minimum ERR by evaluating all $\mathbf{m}_{i}$ in the dataset. However, this is not realistic because $n$ is very large and the cost for evaluating Eq. (1) is non-negligible. On the other hand, there are approaches to efficiently evaluate the properties of deterministic problems by statistical modeling ${ }^{1}$. In this study, we combine such approach with deep learning to reduce the large computational cost.

${ }^{1}$ As an example of such approach, instead of computing the characteristics of the whole matrix directly, there are many methods for investigating the characteristics of 
Firstly, we can consider a random sampling, $n_{0}(\ll n)$, of the dataset with numerous candidate solutions to generate a subspace, in which the problem solution is largely influenced (here, we define datase $_{0}$ as a set of $n_{0}$ candidate solutions randomly chosen from the dataset, (Algorithm 1 line 2)). However, the relation between $\mathbf{m}_{i}$ and $E R R\left(\mathbf{m}_{i}\right)$ is often complicated and thus expressing this relation in explicit form is difficult. Therefore, we use deep learning to obtain this relation. We compute $\operatorname{ERR}\left(\mathbf{m}_{i}\right)$ for all samples $\mathbf{m}_{i}$ in dataset d $_{0}$ using Eq. (1) (Algorithm 1 line 3) and identify the relationship between $\mathbf{m}_{i}$ and $\operatorname{ERR}\left(\mathbf{m}_{i}\right)$ using deep learning (Algorithm 1 line 4). Since $n_{0}$ cannot be large because of the cost of Eq. (1) and that the relationship between $\mathbf{m}_{i}$ and $\operatorname{ERR}\left(\mathbf{m}_{i}\right)$ is complex, we cannot expect enough information (i.e., enough number of modes) required to train a regression model on the whole parameter space of $\mathbf{m}_{i}$. Thus, we generate a classifier, $D L_{a}$, that c lassifies $\mathbf{m}_{i}$ in to several groups based on the value of $E R R$. Since $D L_{a}$ can consider the few effective m odes w ith o nly the few s amples, w e c an expect the training cost of $D L_{a}$ to be negligible. Next, we apply $D L_{a}$ to the dataset to classify all $\mathbf{m}_{i}$ in the dataset. We set $n_{1}(\ll n)$ samples of $\mathbf{m}_{i}$ in the classification group with the smallest $E R R$ to dataset $_{1}$ (Algorithm 1 line 5). This cost is also negligible as $D L_{a}$ is only used for inferencing. Next, we evaluate Eq. (1) for all $\mathbf{m}_{i}$ in dataset $_{1}$ to obtain $\operatorname{ERR}\left(\mathbf{m}_{i}\right)$ (Algorithm 1 line 6). Since the samples in dataset $_{1}$ is selected by a classifier, we can expect a reduction in ERR when compared with that of dataset $t_{0}$. Finally, we train a regression model $D L_{b}$ that outputs ERR for the given $\mathbf{m}_{i}$ (Algorithm 1 line 7). Herein, we use data of dataset $_{0}$ and dataset d $_{1}$ with small $\operatorname{ERR}\left(\mathbf{m}_{i}\right)$ for training. The training cost of $D L_{b}$ is expected to be negligible due to the limited number of samples and the number of effective modes. When compared with a regression model trained on broad-range data, we expect a higher accuracy of $D L_{b}$ in the narrow-range where it is trained on (i.e., region with small $E R R$ ). Using $n_{m}$ samples of $\mathbf{m}_{i}$ with the smallest ERR in dataset $t_{0}$ and dataset $_{1}$, we apply the Karhunen-Loeve expansion based on the subspace method [6] to obtain

$$
\mathbf{m}^{\text {coarse }}=\mathbf{m}^{\text {ave }}+\sum_{j=1}^{n_{m}-1} \alpha_{j} \mathbf{m}_{j}^{\eta},
$$

where, $\mathbf{m}^{\text {ave }}$ denotes the average of $\mathbf{m}_{i}\left(i=1,2,3, \ldots, n_{m}\right)$, and $\mathbf{m}_{j}^{\eta}$ denotes the eigenvector of the covariance matrix of set of vectors $\mathbf{m}_{i}$ (Algorithm 1 line 8$) . \alpha_{j}\left(j=1,2,3, \ldots, n_{m}-1\right)$ is chosen such that $E R R\left(\mathbf{m}^{\text {coarse }}\right)$, which is evaluated by $D L_{b}$, becomes a minimum. This distribution $\mathbf{m}^{\text {coarse }}$ is expected to be a good initial parameter as it includes characteristics of $n_{m}$ samples of $\mathbf{m}_{i}$ with small $E R R$. Finally, using a gradient-based method, we refine $\mathbf{m}^{\text {coarse }}$ on a small-scale computing environment to obtain $\mathbf{m}^{\text {fine }}$ within the available time frame and computational resources (Algorithm 1 line 9).

Because the cost for training and inferencing of $D L_{a}$ and $D L_{b}$ and the cost for the subspace method is negligible and that $n_{0}$ and $n_{1}$ computations of Eq. (1) can be performed in parallel on a supercomputing environment, $\mathbf{m}^{\text {coarse }}$ can be obtained in the time

a matrix via random sampling (e.g., [4, 5]). For example in [5], singular-value decomposition was computed via random sampling instead of directly performing singularvalue decomposition on the target matrix, which enables matrix character estimation with smaller number of samples than the matrix size. required for computing Eq. (1) twice from the users viewpoint. In an environment where real-time data is acquired via high-speed networks, the developed method is expected to be useful considering the need for approximate solutions within a limited time. As it is often extremely inefficient to conduct parameter optimization of problems with bad convergence characteristics without prior information, using the multi-step method for generating a good initial solution is expected to be useful for general optimization problems. Even when solutions can be obtained using a gradientbased method, our approach, which comprises small computation units, is robust and easy to implement when compared with other approaches using many compute nodes in parallel for solving a single gradient-based optimization problem. This feature is expected to be effective when targeting geologically distributed supercomputing resources, such as the cloud.

\section{NUMERICAL EXPERIMENT}

There are many internal-structure estimation problems using largescale wave data measured with many observation equipment located outside of the structure, e.g., biomedical imaging [7, 8], resource exploration [9], crust structure imaging [10, 11], and nondestructive inspection of civil infrastructure [12]. In this section, we demonstrate the effectiveness of the developed method by conducting internal-structure estimation based on wave propagation phenomena using a publicly available geometry data set.

We estimate the distribution of hard material in a cubic domain $\left(-0.64 \leq x_{1} \leq 0.64 \mathrm{~m},-0.64 \leq x_{2} \leq 0.64 \mathrm{~m},-0.64 \leq x_{3} \leq 0.64\right.$ $\mathrm{m})$ with linear elastic material property (primary wave velocity $1.7 \mathrm{~m} / \mathrm{s}$, secondary wave velocity $1.0 \mathrm{~m} / \mathrm{s}$, and density $1.0 \mathrm{~kg} / \mathrm{m}^{3}$ ) (Fig. 2). The cube is fixed at eight vertices. The forward analysis corresponding to Eq. (1) for a given parameter $\mathbf{m}$ representing the distribution of hard material is solved using

$$
\left(c_{i j k l}(\mathbf{m}) u_{k, l}\right)_{, i}=\rho \ddot{u}_{j},
$$

where $c_{i j k l}, u_{j}, \rho,()$ and ( ),j denote heterogeneous elastic tensor, displacement in $j$ direction, density, second order time differentiation, and spatial differentiation in the $j$ direction, respectively. We input $5 \mathrm{~Hz}$ sine waves in the $x_{1}$ direction at $N_{\text {input }}=4$ points at $\left(x_{1}, x_{2}, x_{3}\right)=( \pm 0.32 \mathrm{~m}, \pm 0.32 \mathrm{~m}, 0.64 \mathrm{~m})$. Observation points are located as two-dimensional arrays on each of the six faces of the cubic domain $\left(x_{i}=0.12 j \mathrm{~m}(j=-4,-3,-2, \ldots, 4)\right)$ and displacement in 3 directions $\left(\left\{u_{1}, u_{2}, u_{3}\right\}\right)$ are observed at each point. Thus, the total number of observation points becomes $N_{\text {obs }}=9 \times 9 \times 6=486$, while the total number of observation channels becomes $486 \times 3=$ 1458. ERR is defined as

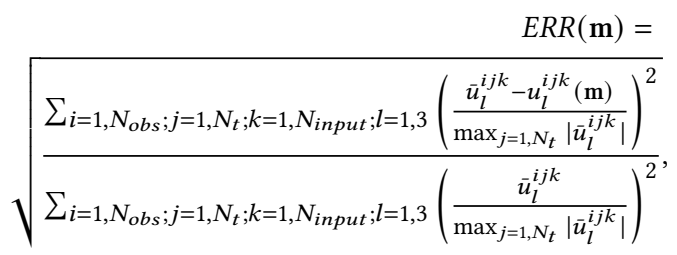

where $N_{t}$ denotes the number of time steps and $\overline{\mathbf{u}}=\mathbf{f}\left(\mathbf{m}^{r e f}\right)$.

We disctretize the domain using voxels and estimate the material property of each voxel. This approach is often used in optimization problems (e.g., [13]). The Young's modulus of the base 


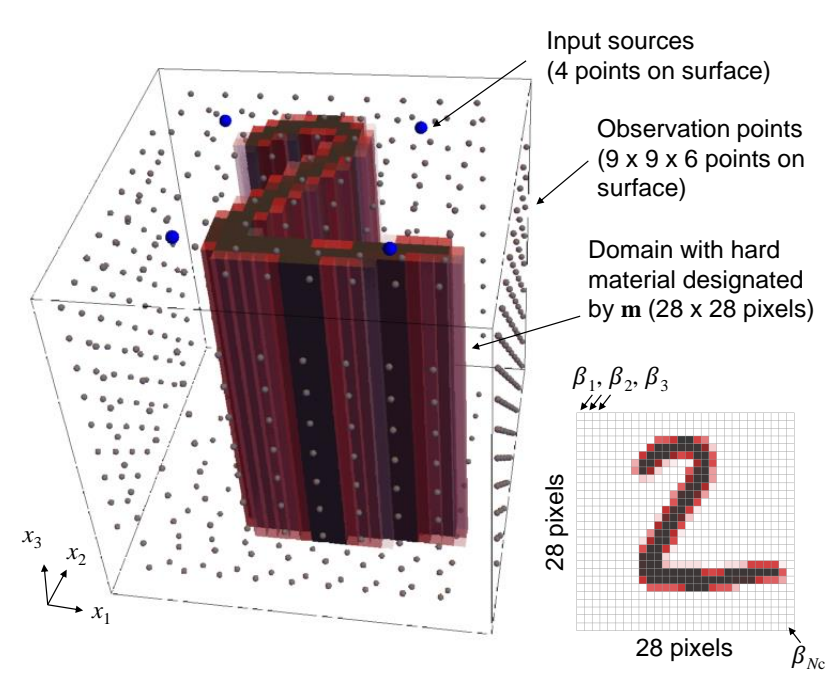

Figure 2: The problem setting of the numerical experiment. Blue dots and gray dots indicate the positions of the input and the observation points, respectively. White-to-dark red color indicates the stiffness of the material, where white and dark red indicate soft and hard materials, respectively. Distribution of the hard material is denoted by $m$ consisting of $28 \times 28$ components.

material is denoted by $E$; Young's modulus of the hard material is given by $\left(1+3 \beta_{i}\right) E$ with $0 \leq \beta_{i} \leq 1$. Here, $\beta_{i}$ are given in $28 \times$ 28 structured points in the $x_{1}, x_{2}$ directions with spacing $d s=0.04$ $\mathrm{m}$ in the following domain: $-0.56 \leq x_{1} \leq 0.56 \mathrm{~m},-0.56 \leq x_{2} \leq$ $0.56 \mathrm{~m},-0.60 \leq x_{3} \leq 0.60 \mathrm{~m}$. The same material property is extruded in the $x_{3}$ direction as shown in Fig. 2. These parameters $\beta_{i}$ composes the parameter vector $\mathbf{m}=\left\{\beta_{1}, \beta_{2}, \beta_{3}, \ldots, \beta_{N_{c}}\right\}$ with $N_{c}=28 \times 28=784$. For the reproducibility of the numerical experiment, we define the distribution of the hard material using the MNIST database [14], which is a database of handwritten numbers "0"-"9" given in $28 \times 28$ pixels. Here, we use "2" of the test data in MNIST to represent $\mathbf{m}^{\text {ref }}$ (Fig. 3a). We generated a dataset of $18831 \times 25=470775(=n)$ samples by 18831 " 1 ," "2," and " 3 "s in the training data of MNIST moved by $0, \pm 1$, and \pm 2 pixels, respectively, in the $x_{1}$ and $x_{2}$ directions. As handwritten numbers are used, we can see that dataset is diverse (Fig. 4); hence, simple sampling of the data would not yield good solutions.

We first try to solve the optimization problem based on conventional methods. A standard method is to use L1 regularization as

$$
E R R^{\prime}=E R R+\lambda \sum_{i=1, N_{c}}\left|\beta_{i}\right|,
$$

and minimize $E R R^{\prime}$ with gradient based methods such as FISTA [15]. Using $\mathbf{m}=\mathbf{0}$ for the initial solution as it is difficult to set a proper initial solution reflecting prior information in the conventional method, we tried to obtain good solutions by changing the $\mathrm{L} 1$ regularization parameter, etc. However, the solutions were trapped in local minimums; thus, we can only obtain poor results

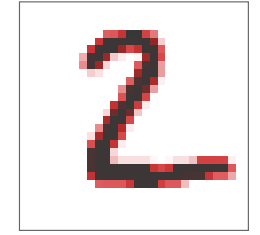

a) $\mathbf{m}^{r e f}(0.0)$

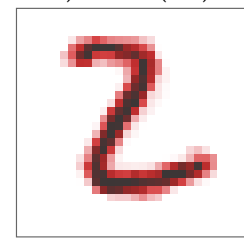

c) $\mathbf{m}^{\text {coarse }}(0.295)$

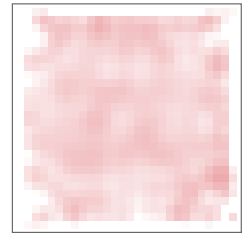

b) $\mathbf{m}^{\text {gradient }}(0.773)$

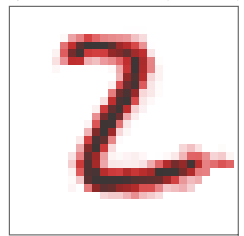

d) $\mathbf{m}^{\text {fine }}(0.238)$

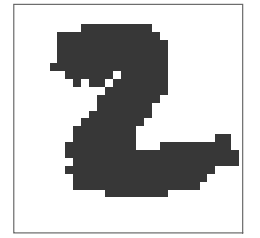

e) Refinement range
Figure 3: Results of the optimization. The numbers in brackets denote values of $E R R$. a) Reference distribution $\mathbf{m}^{\text {ref }}$; b) Result of gradient-based optimization $\mathbf{m}^{\text {gradient }}$ (obtained using FISTA with $\lambda=0.05, n_{\text {iter }}=38$, and initial parameter $\mathrm{m}=0$ ); c) Results of the first step of the fast multi-step optimization with deep learning $\mathrm{m}^{\text {coarse }}$; ) Final results of the fast multi-step optimization with deep learning $\mathbf{m}^{\text {fine }}$ (obtained using FISTA with $\lambda=0.05, n_{\text {iter }}=67$, and initial parameter $\mathrm{m}^{\text {coarse }}$ ); e) Optimization search range using FISTA process of second step in the fast multi-step optimization with deep learning.

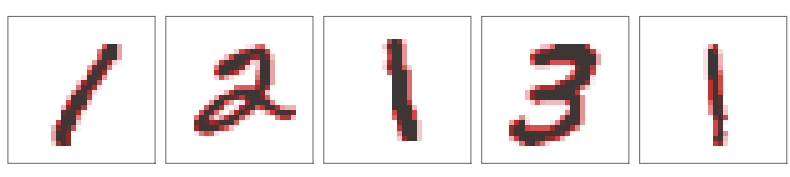

Figure 4: Examples of handwritten " 1 ," “ 2 ," and “ 3 "s in MNIST. Various shapes of " 1 ," " 2 ," and " 3 "s are part of the data set.

(the best of the trials is shown in Fig. 3b). Thus, although it is possible to reduce time of the optimization computation using fast wave propagation methods using large number of compute nodes in parallel (e.g., SC18 Gordon Bell Prize Finalist solver [2] scalable up to full Summit [3]), it is difficult to obtain reasonable solutions for difficult optimization problems such as the problem targeted in this study when using conventional optimization methods. Furthermore, the problem sizes are often too small for efficient computation on very large number of compute nodes, and thus the acceleration is limited even for easier optimization problems. Thus, a fast and efficient method capable of breaking through this situation has been anticipated.

We conduct a parameter optimization using the developed method. We generate dataset $_{0}$ by randomly extracting $1024\left(=n_{0}\right)$ samples from the dataset (Algorithm 1 line 2). The minimum and maximum values of $\operatorname{ERR}\left(\mathbf{m}_{i}\right)$ for $\mathbf{m}_{i}$ in dataset $_{0}$ evaluated using Eq. (4) were 0.471 and 1.012, respectively (Algorithm 1 line 3 ). We equally divide the range between the maximum and minimum values of $E R R$ 


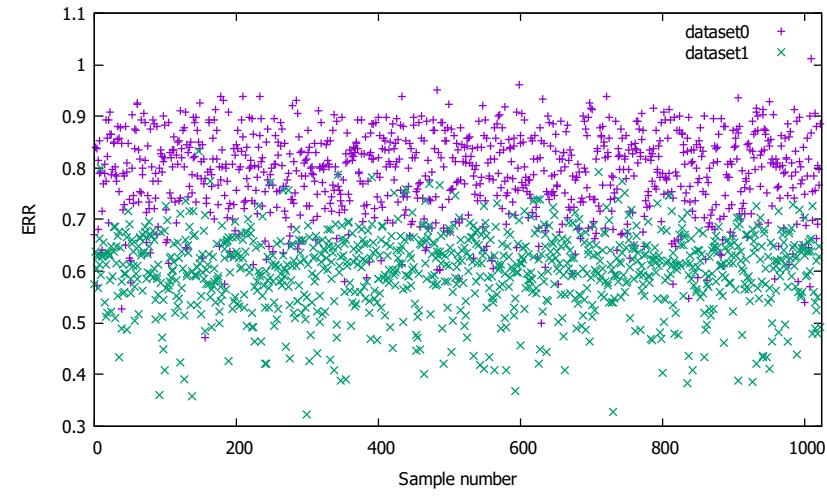

Figure 5: Distribution of $E R R$ for dataset $t_{0}$ and dataset $_{1}$. We can observe that the classification by $D L_{a}$ resulted in a lower $E R R$ compared to the random sampling.

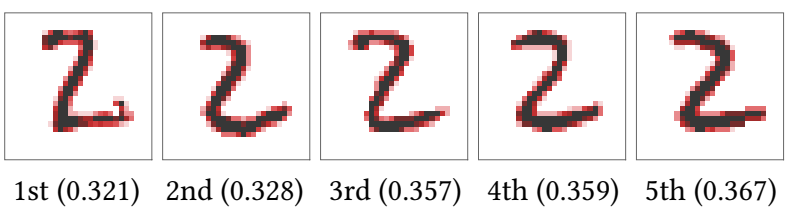

Figure 6: Distribution of $\mathrm{m}$ with the smallest $E R R$ in dataset $_{1}$. The numbers in brackets indicate values of $E R R$.

to make 10 groups, and the data of $\operatorname{ERR}\left(\mathbf{m}_{i}\right)$ in datase $_{0}$ is utilized to generate a classifier with an input vector of $28 \times 28=784$ values and output of classification of $\operatorname{ERR}\left(\mathbf{m}_{i}\right)$ into 10 groups. Following studies on deep learning for recognition of MNIST data, we generate a classifier $D L_{a}$ using a fully connected 784-16-16-16-16-10 feed-forward network (a softmax function is used for the output layer and ReLU functions are used for the other layers) by using 904 samples for training and 120 samples for test data (Algorithm 1 line 4). Herein, we applied TensorFlow [16] and Keras [17] (optimizer $=$ Adam, epochs $=100$, batch size $=200$ ) for training. The cost of constructing $D L_{a}$ is negligible. An application of $D L_{a}$ to all the samples in the dataset ( $n=470775$, which is also a negligible cost), leads to 1543 samples in the smallest ERR group. We set dataset t $_{1}$ by randomly extracting $1024\left(=n_{1}\right)$ samples out of the 1536 samples generated by removing the overlap between datase $_{0}$ and the 1543 samples in the smallest $E R R$ group (Algorithm 1 line 5). Computation of $\operatorname{ERR}\left(\mathbf{m}_{i}\right)$ using Eq. (4) for all the samples in dataset $_{1}$ lead to minimum and maximum ERR of 0.321 and 0.832 , respectively (Algorithm 1 line 6). When the distribution of $\operatorname{ERR}\left(\mathbf{m}_{i}\right)$ for dataset $t_{0}$ and dataset $_{1}$ are compared, we observed that good candidate solutions are extracted by the classifier (Fig. 5). Fig. 6 depicts the five samples with the smallest $\operatorname{ERR}\left(\mathbf{m}_{i}\right)$ in dataset $_{1}$. We can see that good candidate solutions $\mathbf{m}_{i}$ are selected despite the differences in the right hand side of the distribution.

Next we obtain $\mathbf{m}^{\text {coarse }}$. Following work on deep learning for recognition of MNIST data, we generate a regression model, $D L_{b}$, using a fully connected 784-128-16-16-16-16-16-8-1 feed-forward

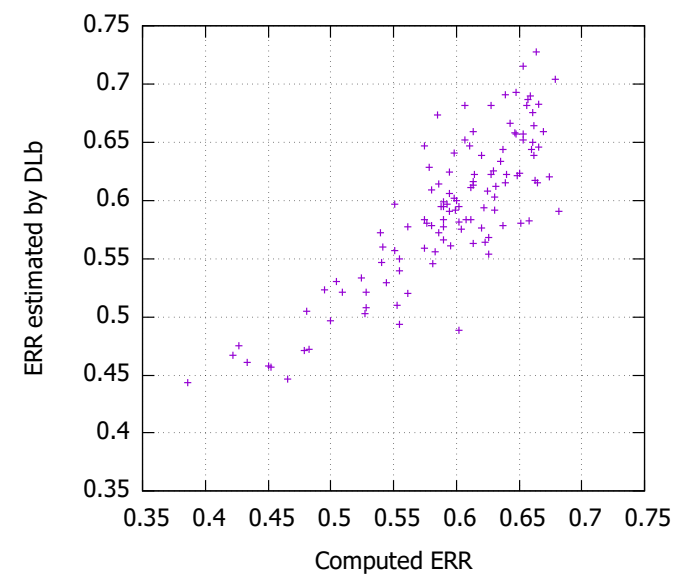

Figure 7: Accuracy of $D L_{b}$. The horizontal axis represents the actual value of $\operatorname{ERR}\left(\mathbf{m}_{i}\right)$, while the vertical axis represents the estimated $E R R$ using $D L_{b}$ for 120 test data. We can see that the estimated value is close to the correct values, and thus the estimation accuracy of $D L_{b}$ is good.

network (ReLU functions are used for all layers) with 1024 samples with smallest $\operatorname{ERR}\left(\mathbf{m}_{i}\right)$ in dataset $_{0}$ and dataset dAlgorithm $1_{1}$ line 7). Herein, 904 samples were utilized for training while 120 samples were used for test data using TensorFlow [16] and Keras [17] (optimizer $=$ Adam, epochs $=200$, batch size $=200$ ). Fig. 7 shows the distribution of $E R R$ obtained by inputting $\mathbf{m}_{i}$ of test data to $D L_{b}$ when compared with the actual $E R R\left(\mathbf{m}_{i}\right)$. We can see that relatively accurate results are obtained within the range of the training data. We generate an initial solution $\mathbf{m}^{\text {coarse }}$ using the five samples with the smallest ERR in dataset $_{0}$ and dataset t $_{1}$ (Fig. 6) by applying $D L_{b}$ on Eq. (2) (Algorithm 1 line 8). In this case, $\alpha_{i}(i=1,2,3,4)$ must be identified; thus, we evaluate $E R R$ using $D L_{b}$ for all cases of $\alpha_{i}(i=1,2,3,4)$ within the range of -5 to 5 using a pitch of 0.25 . This gives the minimum ERR for the case of $\alpha_{1}=-1.0, \alpha_{2}=-0.5, \alpha_{3}=-0.25$, and $\alpha_{4}=0.75$. We apply this set of $\alpha_{i}$ to obtain $\mathbf{m}^{\text {coarse }}$ (Fig. 3c). We can see that the developed method combining conventional method with deep learning is effective as only 2048 samples out of the 470775 samples of dataset with prior information was used in the two-step sampling (sampling ratio was $0.00435(=2048 / 470775))$ for estimating $\mathbf{m}^{\text {coarse }}$ that is close to $\mathbf{m}^{r e f}$. Although the number of parameter sets, in this case, is $((5-(-5)) / 0.25+1)^{4}=2825761$, the cost of evaluating the minimum $E R R$ is negligible since $D L_{b}$ is applied for inference.

Finally, we refine the solution within the available time and computational resources using $\mathbf{m}^{\text {coarse }}$ as the initial solution to obtain the final result $\mathbf{m}^{\text {fine }}$ (Algorithm 1 line 9). Herein, we use FISTA to reduce ERR using regularization in Eq. (5). Fig. 3d shows $\mathrm{m}^{\text {fine }}$ obtained by 67 FISTA iterations. Here, we set the search range of the solution based on the range of solutions of the 25 samples with the smallest $E R R\left(\mathbf{m}_{i}\right)$ in dataset $_{1}$ (Fig. 3e). We observe that $E R R$ reduced from $0.295\left(\mathbf{m}^{\text {coarse }}\right)$ to $0.238\left(\mathbf{m}^{\text {fine }}\right)$, and the distribution on the right-hand side became closer to $\mathrm{m}^{r e f}$. This shows that the refinement of a good initial solution $\mathbf{m}^{\text {coarse }}$ leads 
to a better solution following finer details of the reference solution. If the number of iterations required for refinement from $\mathbf{m}^{\text {coarse }}$ to $\mathbf{m}^{\text {fine }}$ is large, we can extract the relationship between gradientbased optimization meta-parameters and optimization convergence characteristics using deep learning, and use the extracted information for accelerating solution refinement.

In summary, $\mathbf{m}^{\text {coarse }}$ can be obtained in the time of conducting two forward analyses, and $\mathbf{m}^{\text {fine }}$ can be obtained in 67 iterations, with better solutions when compared with the optimization results without using prior information. Although the number of forward analysis iterations is small, the time required for conducting this optimization depends on the time required per the forward analysis of Eq. (3). Together with the analysis speed, portability is important when considering using the method on various available computer resources. Thus, in this study, we use OpenACC [18] to port a CPU-based forward analysis code to GPU environment, and conduct analysis on the AI Bridging Cloud Infrastructure (ABCI) [19] of the National Institute of Advanced Industrial Science and Technology as an example of resource mimicking a cloud-computing environment. As demonstrated in the appendix section, one case of the forward analysis took $33.0 \mathrm{~s}$ on one GPU. Since there was no communication between the GPUs, nearly ideal weak scaling of up to 1024 GPUs was obtained even on the ABCI system, where inter-node bandwidth reduces by three-fold when crossing compute racks. Thus, the developed algorithm can be used to conduct fast parameter optimization on computational resources with relatively low inter-node communication bandwidth and localized data.

\section{CONCLUDING REMARKS}

This study developed a fast multi-step optimization method using deep learning for use on data-centric supercomputing resources. By effective use of prior information by deep learning, approximate solutions can be obtained in a short time, and gradient-based optimization methods can be used to further improve the accuracy. This method can change the situation where large-scale supercomputing resources cannot be utilized for optimization problems due to dependencies and strong scaling limits, and it is expected to be specifically effective for systems with many compute nodes but very limited communication capability (e.g., edge computing resources in BDEC environment). Although we demonstrated our method on an internal structure estimation problem, this method can be applied for other optimization problems with prior information. This approach of using prior information is expected to be useful for various problems in the current society, where fast optimization is required in an environment in which large observational data is available via IoT and 5G networks and large-scale but distributed computer resources are available.

\section{APPENDIX}

We discretize Eq. (3) using the linear voxel finite-element method in space and the finite-difference method in time as follows:

$$
\mathbf{M} \frac{\mathbf{u}_{n}-2 \mathbf{u}_{n-1}+\mathbf{u}_{n-2}}{d t^{2}}+\mathbf{C} \frac{\mathbf{u}_{n-1}-\mathbf{u}_{n-2}}{d t}+\mathbf{K} \mathbf{u}_{n}=\mathbf{0},
$$

where $\mathbf{M}, \mathbf{C}$, and $\mathbf{K}$ denote mass, Rayleigh damping, and stiffness matrices, respectively. $d t$ denotes time increment and $\mathbf{u}_{i}$ denotes the displacement at time step $i$. Element damping matrix $\mathbf{C}_{e}$ is calculated using the element mass matrix, $\mathbf{M}_{e}$, and the element stiffness matrix, $\mathbf{K}_{e}$, as $\mathbf{C}_{e}=\alpha \mathbf{M}_{e}+\beta \mathbf{K}_{e}$. Here, $\alpha$ and $\beta$ are obtained by solving the following least-squares equation:

$$
\operatorname{minimize}\left[\int_{f_{\min }}^{f_{\max }}\left(h-\frac{1}{2}\left(\frac{\alpha}{2 \pi f}+2 \pi f \beta\right)\right)^{2} \mathrm{~d} f\right] \text {, }
$$

where $f_{\max }, f_{\min }$, and $h$ denote the maximum and minimum target frequencies and the damping ratio, respectively. By applying lumped mass approximation to $\mathbf{M}$ in Eq. (6), we can explicitly compute $\mathbf{u}_{n}$ as follows:

$$
\begin{array}{r}
\mathbf{u}_{n}=2 \mathbf{u}_{n-1}-\mathbf{u}_{n-2}+\mathbf{M}^{-1} \\
\left\{\mathbf{K} d t\left(\beta \mathbf{u}_{n-2}-(\beta+d t) \mathbf{u}_{n-1}\right)+\mathbf{M} \alpha d t\left(\mathbf{u}_{n-2}-\mathbf{u}_{n-1}\right)\right\} .
\end{array}
$$

In this study, we use $f_{\max }=1.0 \mathrm{~Hz}, f_{\min }=0.05 \mathrm{~Hz}$, and damping factor $h=0.005$. By considering the Courant condition, we use an element size of $0.01 \mathrm{~m}$ and time step increment of $d t=0.0005 \mathrm{~s}$ for computing the problem of $2 \mathrm{~s}$ duration. The problem becomes 128 elements (129 nodes) in the $x_{1}, x_{2}, x_{3}$ directions, leading to 2097152 elements and 2146689 nodes with $N_{t}=4000$-time steps. All computation is performed in double-precision to ensure computational accuracy.

We consider developing an implementation of Eq. (7) for various computing resources consisting of CPUs and GPUs. We first develop an efficient CPU code and port it to the GPU environment using OpenACC. Since the current computational resources have relatively less memory capacity compared to its arithmetic capabilities, it is often difficult to explicitly allocate the global matrix of finite-element methods on fast memory. Thus, for computation of the matrix-vector product $\mathrm{y} \Leftarrow \mathrm{Ax}$, we use the element-byelement method [20], which is a type of matrix-free matrix-vector product $\left(\mathrm{y} \Leftarrow \sum_{e} \mathbf{A}_{e} \mathbf{x}_{e}\right.$ ), where subscripts $e$ denotes values for each element. Since data recurrence is involved in computing $\mathbf{y}$, care is required when conducting parallel computation. We apply coloring with OpenMP [21] for thread allocation on CPUs, and we can also use hardware accelerated atomics to obtain a reasonable performance on GPUs. All computation is conducted on GPUs and only outputting of results is conducted on the CPU for the GPU implementation. The program is designed such that small cost is required in developing multi-platform code (i.e., directives, such as, designating the timing of host-device data transfer and designating where to use atomics are inserted in the CPU code).

Table 1 shows the performance using one ABCI node. Here, we use 2 twenty-core Intel Xeon Gold 6148 CPUs or 4 NVIDIA Tesla V100 GPUs for computing 4 sets of $\operatorname{ERR}\left(\mathbf{m}_{i}\right)$. We can see that the elapsed time ratio between the CPU and GPU implementation was $1: 12.7$. This is a reasonable ratio considering that the hardware peak FLOPS ratio between the CPUs and GPUs of an ABCI node is 3.07 TFLOPS (CPUs, 1.53 TFLOPS $\times 2$ ) : 31.2 TFLOPS (GPUs, 7.8 TFLOPS $\times 4)=1: 10.2$, and hardware peak memory bandwidth is $256 \mathrm{~GB} / \mathrm{s}(\mathrm{CPUs}, 128 \mathrm{~GB} / \mathrm{s} \times 2)$ ) $3600 \mathrm{~GB} / \mathrm{s}(\mathrm{GPUs}, 900 \mathrm{~GB} / \mathrm{s} \times$ 4) $=1: 14.1$. The measured performance per GPU was 1.41 TFLOPS $(273 \mathrm{~GB} / \mathrm{s})$, which is considered reasonable for a low-ordered finiteelement method. From Table 2, we can observe that the computation of 1024 sets of $E R R\left(\mathbf{m}_{i}\right)$ using 256 nodes (1024 GPUs) finished in almost the same time required for computing 4 sets using one 
Table 1: Elapsed time for solving four sets of $\mathrm{m}_{i}$ using CPU/GPU resources on one node of ABCI.

\begin{tabular}{lrr} 
& Elapsed time & Speed-up ratio \\
\hline CPU: 10 OpenMP threads $\times 4$ sets & $421.2 \mathrm{~s}$ & 1 \\
GPU: 1 V100 GPU $\times 4$ sets & $33.0 \mathrm{~s}$ & 12.7 \\
\hline
\end{tabular}

Table 2: Weak scaling of the solver on GPU resources of ABCI. Elapsed time measured for solving one sample of $\mathbf{m}_{i}$ per GPU.

\begin{tabular}{rrrr} 
Number of nodes & Number of GPUs & Elapsed time & Scalability \\
\hline 1 & 4 & $33.0 \mathrm{~s}$ & NA \\
256 & 1024 & $34.0 \mathrm{~s}$ & $97.0 \%$ \\
\hline
\end{tabular}

node (4 GPUs). This shows that the algorithm is capable of attaining performance according to the capability of the computational resource used.

As gradient computation is added, the computation of a FISTA iteration for obtaining $\mathbf{m}^{\text {fine }}$ required $63.8 \mathrm{~s}$ using a V100 GPU. However, this is only performed using one GPU and thus comprises a small cost within the whole computation; the number of iterations for obtaining $\mathbf{m}^{\text {fine }}$ can be chosen according to the available time.

\section{ACKNOWLEDGMENTS}

We acknowledge support from the Post K computer project (Priority Issue 3 - Development of integrated simulation systems for hazards and disasters induced by earthquakes and tsunamis: proposal numbers hp180217, hp190177), Japan Society for the Promotion of Science (18H05239, 18K18873, 17K14719), and CART project. Our results were obtained using computational resource of the AI Bridging Cloud Infrastructure (ABCI) at the National Institute of Advanced Industrial Science and Technology.

\section{REFERENCES}

[1] BDEC2: Big Data and Extreme-Scale Computing 2, [Online]. https://www.exascale.org/bdec/

[2] T. Ichimura, K. Fujita, T. Yamaguchi, A. Naruse, J. C. Wells, T. C. Schulthess, T. P. Straatsma, C. J. Zimmer, M. Martinasso, K. Nakajima, M. Hori, L. Maddegedara, A fast scalable implicit solver for nonlinear time-evolution earthquake city problem on low-ordered unstructured finite elements with artificial intelligence and transprecision computing, SC '18 Proceedings of the International Conference for High Performance Computing, Networking, Storage, and Analysis, Article No. 49, 2018.

[3] Summit, [Online] https://www.olcf.ornl.gov/olcf-resources/compute-systems/summit/

[4] N. Halko, P. G. Martinsson, and J. A. Tropp, Finding Structure with Randomness: Probabilistic Algorithms for Constructing Approximate Matrix Decompositions, SIAM Rev., 53(2), pp. 217-288, 2011.

[5] A. Frieze, R. Kannan, S. Vempala, Fast Monte-Carlo algorithms for finding lowrank approximations, Proceedings 39th Annual Symposium on Foundations of Computer Science, pp. 370-378, 1998.

[6] S. Watanabe and N. Pakvasa, Subspace method of pattern recognition, Proc. 1st international conference on pattern recognition, pp. 25-32, 1973.

[7] D. Huang, E. A. Swanson, C. P. Lin, J.S. Schuman, W.G. Stinson, W. Chang, M. R. Hee, T. Flotte, K. Gregory, C. A. Puliafito, et al., Optical coherence tomography, Science, 22 Nov 1991: Vol. 254, Issue 5035, pp. 1178-1181, DOI: 10.1126/science. 1957169

[8] A. C. Kak, M. Slaney, Principles of Computerized Tomographic Imaging, Society of Industrial and Applied Mathematics, 2001.
[9] M. Warner, A. Ratcliffe, T. Nangoo, J. Morgan, A. Umpleby, N. Shah, V. Vinje, I. Stekl, L. Guasch, C. Win, G. Conroy, A. Bertrand, Anisotropic 3D full-waveform inversion, Geophysics, Vol. 78, No. 2, pp. R59-R80, 2013.

[10] M. Rietmann, P. Messmer, T. Nissen-Meyer, D. Peter, P. Basini, D. Komatitsch, O. Schenk, J. Tromp, L. Boschi, and D. Giardini. Forward and adjoint simulations of seismic wave propagation on emerging large-scale GPU architectures, Proceedings of the International Conference on High Performance Computing, Networking, Storage and Analysis (SC'12). IEEE Computer Society Press, Los Alamitos, CA, USA, 2012, Article 38, 11 pages.

[11] A. Gokhberg, A. Fichtner, Full-waveform inversion on heterogeneous HPC systems, Computer \& Geosciences, Vol. 89, pp. 260-268, 2016, DOI: 10.1016/j.cageo.2015.12.013.

[12] C. Maierhofer, Nondestructive Evaluation of Concrete Infrastructure with Ground Penetrating Radar, Journal of Materials in Civil Engineering, Vol. 15, Issue 3 (June 2003), https://doi.org/10.1061/(ASCE)0899-1561(2003)15:3(287)

[13] N. Aage, E. Andreassen, B. S. Lazarov, and O. Sigmund, Giga-voxel computational morphogenesis for structural design, Nature, vol. 550(7674), pp. 84-86, 2017.

[14] Y. LeCun, C. Cortes, C. J. C. Burges, THE MNIST DATABASE of handwritten digits, [Online]. http://yann.lecun.com/exdb/mnist/.

[15] Beck and Teboulle, A fast iterative shrinkage-thresholding algorithm for linear inverse problems SIAM Journal on Imaging Sciences, 2009.

[16] TensorFlow, [Online]. https://www.tensorflow.org/

[17] Keras, [Online]. https://keras.io/

[18] OpenACC, [Online]. https://www.openacc.org/

[19] AI Bridging Cloud Infrastructure, [Online]. https://abci.ai/

[20] J. M. Winget, T. J. R. Hughes, Solution algorithms for nonlinear transient heat conduction analysis employing element-by- element iterative strategies, Computer Methods in Applied Mechanics and Engineering, 52, pp. 711-815, 1985.

[21] OpenMP, [Online]. https://www.openmp.org/ 